\title{
Mapas Conceituais para Avaliação do Ensino- Aprendizagem em uma Disciplina de Engenharia de Transportes
}

\author{
Carlos Alberto Prado da Silva Junior ${ }^{1 *}$, Heliana Barbosa Fontenele ${ }^{1}$, Antônio Nélson \\ Rodrigues da Silva² \\ ${ }^{1}$ Universidade Estadual de Londrina \\ ${ }^{2}$ Escola de Engenharia de São Carlos da Universidade de São Paulo \\ * Autor para correspondência: cprado@uel.br
}

\begin{abstract}
RESUMO
O objetivo principal deste estudo foi realizar uma análise comparativa dos resultados obtidos a partir do uso de duas técnicas diferentes para a avaliação do processo de ensino-aprendizagem em um curso de graduação em Planejamento e Análise de Sistemas de Transportes. A primeira é uma abordagem tradicional, que se baseia essencialmente em provas e testes, enquanto a segunda se vale de mapas conceituais. Os mapas conceituais construídos por cada aluno durante o curso foram cuidadosamente analisados e receberam notas objetivas. A nota final atribuída aos mapas foi então comparada com a média das notas atribuídas aos exames e questionários de cada aluno. Os resultados da comparação sugerem que os mapas conceituais podem auxiliar e aperfeiçoar a avaliação do processo de ensino-aprendizagem. Além disso, os mapas tornaram a avaliação mais abrangente em relação à evolução cognitiva, ao domínio conceitual e de relações conceituais para o caso específico deste estudo. $\mathrm{O}$ estudo conclui que os mapas conceituais constituem uma alternativa potencialmente útil para complementar outras estratégias de avaliação do ensino-aprendizagem.
\end{abstract}

Palavras-chave: Mapas Conceituais; Avaliação; Ensino-Aprendizagem; Engenharia de Transportes.

\begin{abstract}
The main objective of this study was to conduct a comparative analysis of the outcomes obtained with two different techniques used for the assessment of the teaching-learning process in an undergraduate course on Transportation Systems: Analysis \& Planning. The first one is a traditional approach, which is essentially based on exams and quizzes, whereas the second approach is based on concept maps. The concept maps built by each student along the course were thoroughly analyzed and objectively graded. The final grade of the maps was then compared with the average grade attributed to the exams and quizzes of each student. The results of the comparison suggest that concept maps can support and improve the assessment of the teaching-learning process. In addition, the maps also complemented the evaluation regarding aspects of the cognitive evolution, conceptual domain, and conceptual relationships for the case under study. The study concludes that concept maps are a potentially useful alternative for complementing other teaching-learning assessment approaches.
\end{abstract}

Keywords: Concept Maps; Assessment; Teaching-Learning; Transportation Engineering.

\section{Introdução}

Os sistemas de transportes podem produzir fortes impactos na qualidade de vida da população de determinada região. A avaliação desses impactos, que tanto podem ser positivos quanto negativos, depende, em grande medida, do enfoque dado ao processo de análise e planejamento. Nas últimas décadas tem havido uma mudança de paradigma com relação a esse processo, e a prática de prever e simplesmente prover (novas infraestruturas viárias, por exemplo) tem sido substituída por questões de maior amplitude, tais como: planejamento integrado e mobilidade urbana. Sobre essa questão do prever e prover, Goulden et al. (2014) ressaltam o estudo apresentado por Adams (1981) há mais de três décadas. 
Para reforçar essa ideia, Rodrigues da Silva (2010) destaca algumas mudanças na forma como vêm sendo planejados os sistemas de circulação urbanos. Segundo o autor, para que essas mudanças se concretizem, no entanto, o processo de formação dos profissionais (muitos dos quais engenheiros) que irão atuar em questões relativas aos sistemas de transportes e, mais recentemente, em questões mais amplas de mobilidade urbana, também precisa ser revisto. Em consequência disso, a educação em engenharia também precisa passar e vem passando, de forma geral, por uma série de transformações.

Apesar de muitas dessas transformações estarem ligadas a processos mais gerais (como a globalização, por exemplo), do ponto de vista do ambiente de ensino o impacto é mais visível na sala de aula. Tendo em vista que a maior parte da educação formal de engenheiros tradicionalmente se dá em sala de aula, alterar esse ambiente significa, todavia, mudar estruturas consagradas por décadas de uso e acomodação (SILVA JUNIOR et al., 2015). Sendo assim, o objetivo deste estudo, que é parte de uma pesquisa mais ampla, foi estabelecer uma análise comparativa de uma abordagem que mesclou avaliação tradicional (provas e testes) e mapas conceituais, em dois anos letivos distintos (2012 e 2013) em uma disciplina que trata de análise e planejamento de sistemas de transportes.

\section{Mapas Conceituais para Avaliação do Processo de Ensino-Aprendizagem em Engenharia de Transportes}

No atual contexto no Brasil, os profissionais que atuam na área de Engenharia de Transportes são oriundos geralmente dos cursos de Engenharia Civil. Porém, esses cursos muitas vezes não adotam procedimentos que motivem os alunos para a percepção da complexidade do planejamento de transportes. Segundo Silva Junior et al. (2013), a maioria desses cursos não possui, por exemplo, carga horária suficiente para tratar da magnitude do tema. Além disso, a avaliação do processo de ensino-aprendizagem é conduzida de forma tradicional, ou seja, a verificação da aprendizagem se dá predominantemente através de registro de notas (LUCKESI, 2011). Para Corrêa (2009), isso representa apenas uma perspectiva classificatória e não pode ser vista como uma avaliação formativa e inclusiva.

A avaliação formativa pode atuar como subsídio para possíveis intervenções e resolução de equívocos de aprendizagem. Nesse sentido, os mapas conceituais têm desempenhado importante e bem-sucedido papel na avaliação formativa de acadêmicos de diversos cursos de engenharia. Vários estudos apontam que mapas conceituais têm auxiliado professores e alunos na identificação de problemas e na construção de um processo de ensino-aprendizagem mais dinâmico, duradouro e abrangente, conforme Souza e Boruchovitch (2010a) e Silva Junior et al. (2015).

O mapa conceitual é uma ferramenta pedagógica proposta por John Novak na década de 1970. Pode ser usado para representar graficamente conceitos e suas relações. A teoria da aprendizagem significativa, proposta por David Ausubel (AUSUBEL et al., 1980; MOREIRA \& MASINI, 1982; e PALMERO, 2004), é a base para a construção desses mapas (SILVA JUNIOR, 2014). Além disso, os mapas conceituais podem ser utilizados como ferramentas de ensino-aprendizagem e/ou de avaliação. Como ferramenta para avaliação do processo de ensino-aprendizagem, Novak (2003) ressalta que o mapa conceitual construído pelo aluno permite identificar ideias válidas e equívocos sobre determinado conhecimento, favorecendo o acompanhamento dinâmico da avaliação. Com base nisso, é possível estabelecer de fato um processo de avaliação da aprendizagem, ao invés de apenas realizar a verificação da aprendizagem, como salienta Luckesi (2011).

Segundo Silva Junior et al. (2015), o uso de mapas conceituais pode produzir uma avaliação do processo de ensino-aprendizagem mais abrangente, o que permite uma análise da evolução do processo cognitivo. Dessa forma, os mapas possibilitam observar a evolução do domínio conceitual e do domínio de relação conceitual dos alunos. Como resultado, os mapas conceituais podem evidenciar aprendizagem significativa e, consequentemente, 
constituir-se em uma ferramenta para apoiar o processo de avaliação da aprendizagem. Essa foi, em grande medida, a motivação dos referidos autores para a utilização dos mapas como parte das estratégias pedagógicas adotadas para a formação de engenheiros na área de transportes (para mais detalhes, ver SILVA JUNIOR, 2014).

\section{Método}

O objetivo principal dos procedimentos adotados foi acompanhar e avaliar a aquisição de competências e habilidades, por parte dos alunos, em relação aos conteúdos relativos à disciplina que tratava de análise e planejamento de sistemas de transportes. O software IHMC - CmapTools foi utilizado na construção dos mapas conceituais. Os mapas foram elaborados de acordo com Novak (2003) e conforme a teoria da aprendizagem significativa de Ausubel (1980).

Cada aluno que participou do estudo construiu três versões de mapas conceituais: i) a primeira versão, na semana inicial de aulas da disciplina, com o objetivo de identificar os organizadores prévios e de se obter uma versão prévia do mapa; ii) a segunda versão, após metade da carga horária ter sido cumprida e antes do início das atividades de aprendizagem ativa relacionadas à metodologia da problematização (ver BERBEL, 1998, e SILVA JUNIOR, 2014); e iiii) a terceira versão, ao final de todas as atividades da disciplina. Vale ressaltar que os organizadores prévios serviram como referência para a elaboração das aulas expositivas e das atividades que estavam alinhadas com métodos de aprendizagem ativa, tais como: a metodologia da problematização; trabalhos em equipes; e e-learning, por meio do sistema on-line Tidia-Ae.

Para avaliar os mapas, foram estabelecidos os seguintes critérios de análise, adaptados de Moreira e Masini (1982), Souza (2004), Cantú (2005), Ruiz-Moreno et al. (2007), Stange Lopes (2007), Tavares e Luna (2007), Corrêa (2009) e Souza e Boruchovitch (2010a, 2010b e 2010c): características comuns nas versões dos mapas conceituais - CGM; problemas recorrentes - PBR; conceitos introduzidos - COI; importância dos conceitos introduzidos
- ICOI; evolução nas três versões dos mapas conceituais - EVMC; dificuldade para a construção dos mapas conceituais - DCMC; conceitos preservados nas três versões dos mapas conceituais CPMC; e relação entre valores atribuídos ao mapa conceitual final e à média final - RMCMF.

Além de acompanhar a evolução conceitual, o domínio conceitual e o domínio procedimental dos alunos, foi objetivo do método estabelecer uma relação entre a versão final do mapa construído por cada aluno e sua média final na disciplina. Partiu-se do pressuposto de que seria possível, com base nessa comparação, estabelecer uma avaliação do processo de ensino-aprendizagem mais dinâmica e fidedigna. Assim, ao comparar o mapa final de cada aluno com sua média global, seria possível avaliar, de forma mais abrangente, o processo de aquisição de competências e habilidades. Também foi solicitado que os alunos respondessem nove questões referentes à sua percepção com o uso dos mapas conceituais. Essas questões foram aplicadas ao final da disciplina por meio de um questionário on-line.

\section{Resultados}

Os alunos que participaram deste estudo produziram, em momentos distintos dos anos de 2012 e 2013, três versões de mapas sobre o tema geral da disciplina. Além disso, foi solicitada a construção de outros mapas, sobre temas diversos, com a finalidade de familiarizar os alunos com o uso do software CmapTools e o próprio processo de construção de mapas. A seguir serão apresentadas as análises dos mapas conceituais para uma turma de 2012 e outra de 2013, conforme Silva Junior (2014) e Silva Junior et al. (2015). Vale ressaltar que, em 2013, duas turmas distintas da mesma disciplina construíram mapas conceituais. Uma delas, que teve a maioria das aulas teóricas ministradas por outro docente, não foi, no entanto, objeto de análise neste estudo (embora os resultados constem do trabalho de Silva Junior, 2014).

\section{Avaliação dos Mapas Conceituais}

Nas Figuras 1 e 2 tem-se a representação geral da avaliação das três versões dos mapas conceituais 
construídos pelos alunos das turmas de 2012 e de 2013, respectivamente. Várias análises podem ser feitas, a partir dessas figuras, em relação aos aspectos positivos e negativos da avaliação geral dos mapas conceituais. O foco deste artigo, porém, será apenas nos itens de avaliação que apresentaram discrepância visível de um ano para o outro. São eles: COI - Conceitos introduzidos; ICOI - Importância dos conceitos introduzidos; e RMCMF - Relação entre valores atribuídos ao Mapa Conceitual Final e à Média Final.

Para o caso dos critérios COI e ICOI, parece haver uma explicação plausível para a diferença de resultados de um ano para o outro. Em 2012, os alunos introduziram poucos conceitos novos, o que afetou os dois itens de forma negativa. Concluído o período letivo, no entanto, uma análise retrospec- tiva do processo indicou que isso poderia ter sido consequência da lista de conceitos fornecida pelo professor como referência inicial. Por ser muito extensa (continha trinta conceitos), pode ter prejudicado o desempenho dos alunos nos dois itens. A partir dessa constatação, a lista fornecida como referência inicial em 2013 foi sensivelmente menor. Tal mudança parece ter produzido o efeito desejado, uma vez que, no segundo ano, o desempenho dos alunos nos dois itens foi plenamente positivo.

No que diz respeito ao critério RMCMF (Relação entre valores atribuídos ao mapa conceitual final e à média final), podem ser feitas as seguintes considerações para o caso da turma de 2012, conforme a Figura 1: i) em 46\% dos casos, houve uma forte relação entre a avaliação quantitativa atribuída aos

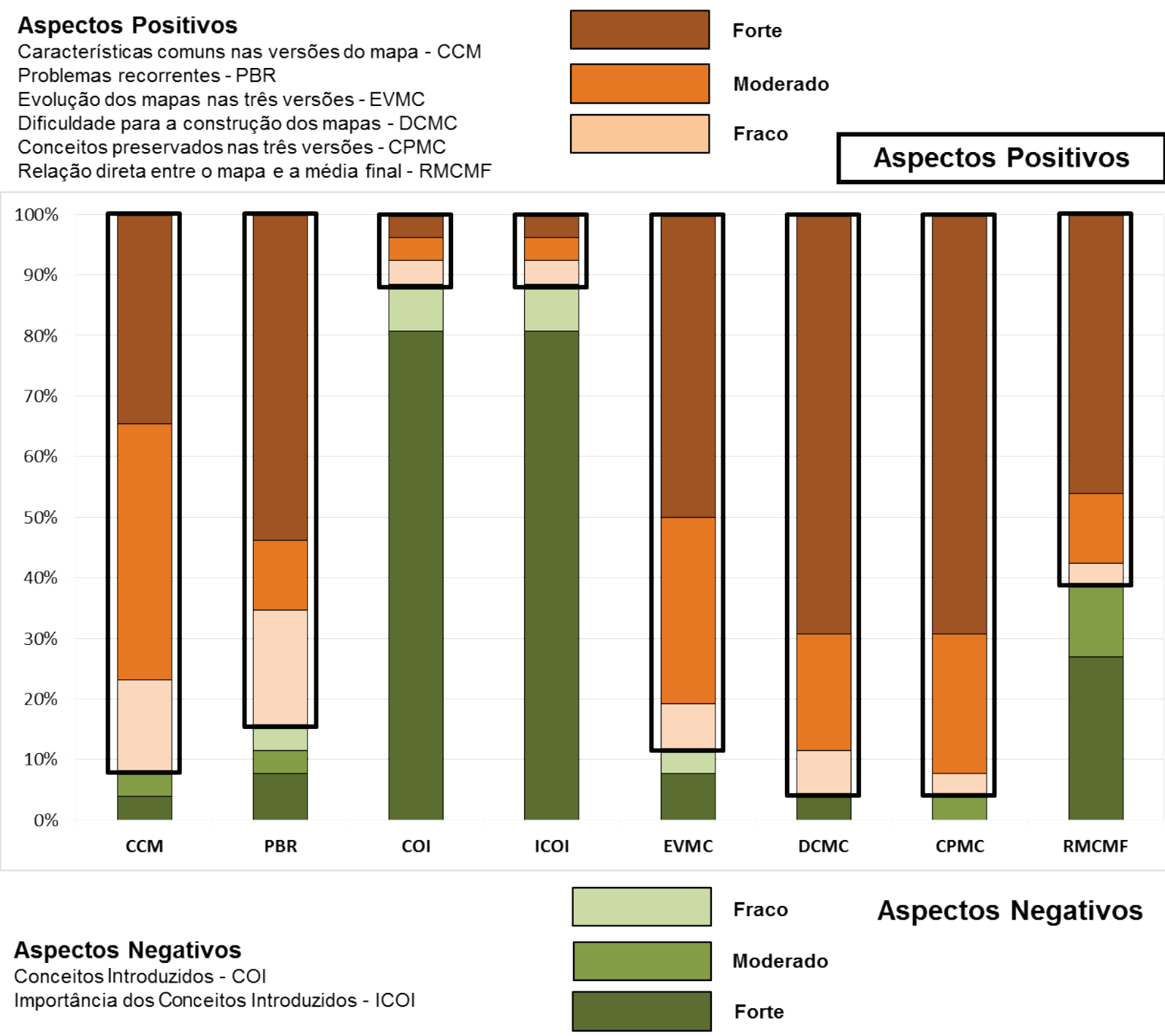

Figura 1 - Avaliação geral dos mapas conceituais - Turma 2012. 
mapas e à média final de cada aluno; ii) em 12\% dos casos, a relação foi moderada; e iii) em $4 \%$ dos casos, foi fraca. Dos $46 \%$ e $12 \%$ dos alunos que tiveram relação forte a moderada entre a avaliação quantitativa dos mapas e a média final, foi constatado que 31\% obtiveram média final ruim, mas construíram bons mapas; 4\% tiveram média final boa e construíram mapas pobres; e 8\% tiveram média final ruim e construíram mapas pobres (informação não explícita na Figura 1).

Essa discordância entre os valores atribuídos ao mapa conceitual final e a média final do aluno identificada nos resultados de 2012 reforça as dúvidas quanto à eficácia de avaliações baseadas apenas em provas e testes de forma tradicional. No entanto, ao analisar os resultados obtidos para o item RMGMF para a turma de 2013 (Figura 2), observa-se que ocorreu relação direta em 100\% dos casos, ou seja, a média final estava muito próxima da nota atribuída ao mapa. Esse resultado suscita novas dúvidas, que podem eventualmente ser assim justificadas para o caso específico deste estudo: i) houve, para a turma de 2013, melhor condução do processo de uso dos mapas conceituais em conjunto com avaliação tradicional; ou ii) havia diferenças significativas entre as duas turmas. Em qualquer dos casos, contudo, parece ficar claro que o uso de mais de um instrumento pode auxiliar o professor na avaliação do processo de ensino-aprendizagem.

\section{Percepção dos Alunos sobre o Uso dos Mapas Conceituais}

A seguir serão apresentados os resultados relativos a um questionário respondido pelos alunos da

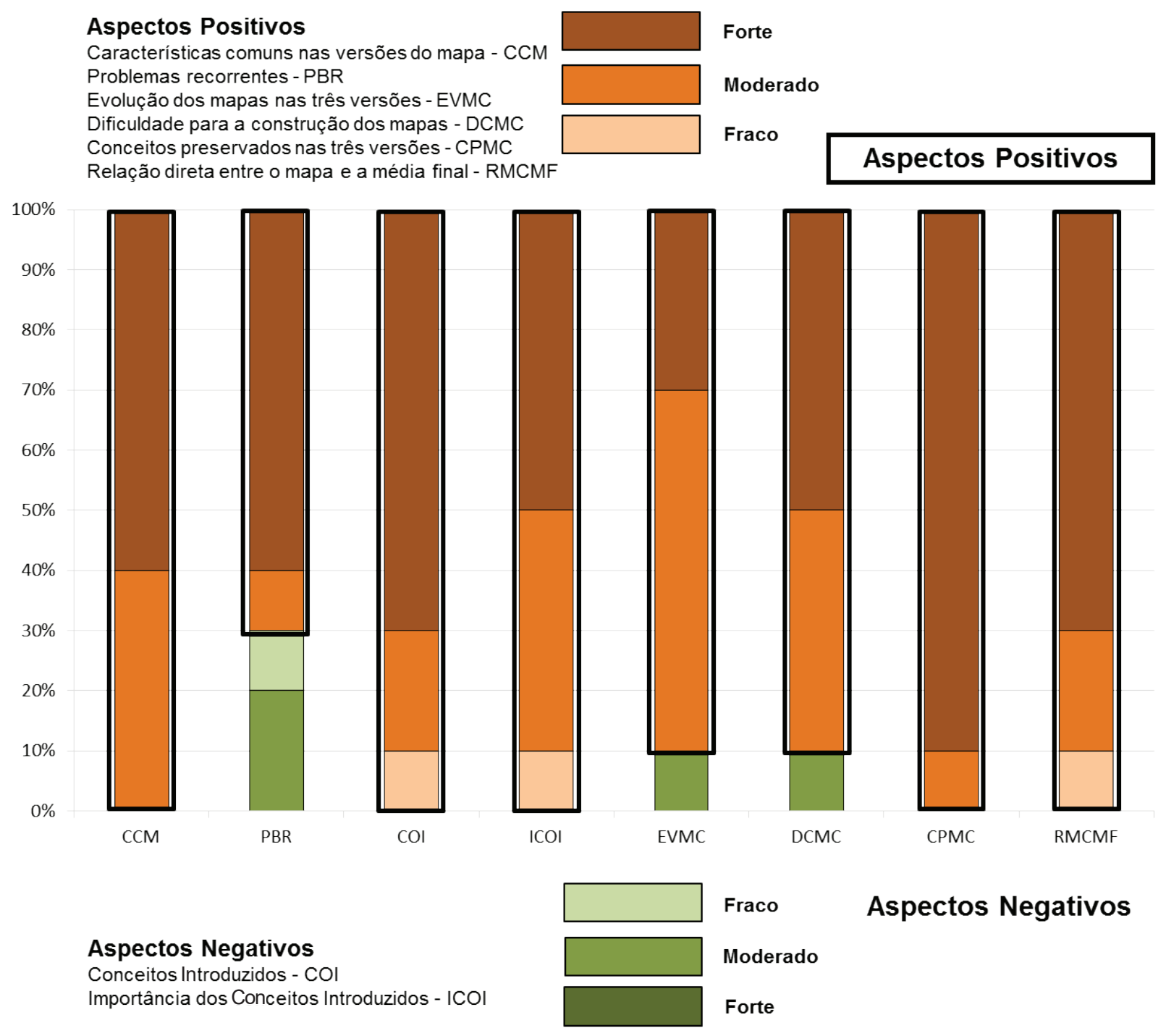

Figura 2 - Avaliação geral dos mapas conceituais - Turma 2 - 2013 
turma de 2012 e turma 2 de 2013. O objetivo desse questionário era obter a percepção deles sobre o uso dos mapas conceituais e do software CmapTools na disciplina de Análise e Planejamento de Sistemas de Transportes. O questionário era composto de nove perguntas diretas, com a possibilidade de comentários. A forma de preenchimento do questionário foi por meio de uma plataforma on-line. As respostas às perguntas do questionário foram divididas em quatro temas: $\imath$ ) confecção dos mapas conceituais; ii) dificuldades para a construção dos mapas conceituais; iiı) mapas conceituais como ferramenta de avaliação; e iv) percepção geral. Neste artigo serão apresentadas as respostas relativas apenas ao item $i$. Para mais informações sobre os demais itens, sugere-se consultar o trabalho de Silva Junior (2014).

Com relação à percepção geral sobre o uso dos mapas conceituais e do software CmapTools

\section{Você utilizou o programa CmapTools para fazer outras atividades além daquelas solicitadas pelo professor?}

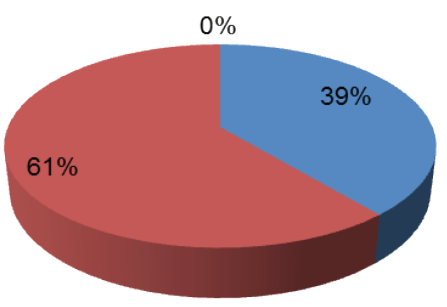

- Sim $\backsim$ Não -Outro

(a)
(Figura 3), têm-se as seguintes análises: ı) 61\% dos alunos não encontraram outros usos para o software (gráfico a); il) 72\% consideraram os mapas conceituais interessantes e úteis (gráfico b); e iii) $67 \%$ dos alunos usariam os mapas conceituais nas demais atividades do curso de Engenharia Civil (gráfico c). Nesse caso foram citados como exemplos: fazer resumos das aulas diárias; sumarizar textos extensos; estudar assuntos teóricos e sem cálculos matemáticos; e estudar para provas e testes. Com base nesse último item, observa-se que alguns alunos perceberam a aplicação dos mapas conceituais como ferramenta de ensino-aprendizagem. Dessa forma, há indícios de que os mapas foram bem aceitos pela maioria dos alunos.

$\mathrm{Na}$ Tabela 2 são apresentados comentários de dois alunos sobre outros usos para os mapas conceituais no curso de Engenharia Civil. Os alunos que apresentaram esses comentários disseram que

\section{De uma forma geral, qual sua opinião sobre os mapas conceituais?}

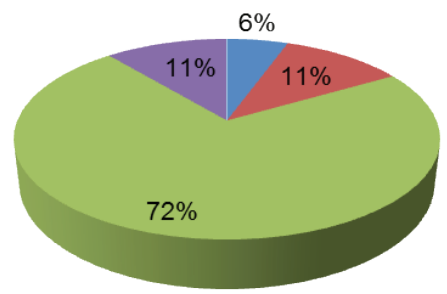

- Útil, mas não interessante

- Nem Interessante e nem útil - Interessante e útil

(b)

\section{Você faria outros usos para o recurso "mapa conceitual" durante o curso de Engenharia Civil?}

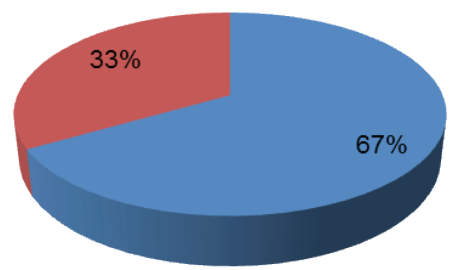

$\square \operatorname{Sim} \backsim$ Não

(c) 


\begin{tabular}{|c|c|c|}
\hline Aluno & \multicolumn{1}{c}{ Comentário } \\
\hline 1 & $\begin{array}{l}\text { "Acredito que seja uma ótima maneira de estudar pra quem não consegue memorizar } \\
\text { conceitos a partir de textos compridos. Pode ser utilizado em qualquer matéria mais } \\
\text { teórica, sem muitos cálculos (exemplo: materiais de construção)." }\end{array}$ \\
\hline 2 & $\begin{array}{l}\text { "Fazer resumos do conhecimento adquirido em sala de aula, de modo que se possa } \\
\text { organizar e hierarquizar os conceitos, e relacioná-los entre si." }\end{array}$ \\
\hline
\end{tabular}

Tabela 2 - Comentários de alunos sobre outros usos dos mapas conceituais.

fariam outros usos para os mapas nas atividades do curso em geral. Mais uma vez, ficam evidentes a importância e a utilidade dessa ferramenta no processo de ensino-aprendizagem.

\section{Conclusões}

Os mapas conceituais mostraram-se viáveis e úteis para a avaliação da aprendizagem dos alunos. Isso pode ser comprovado pela análise conduzida em relação às três versões dos mapas construídos. Foi possível estabelecer uma comparação da avaliação quantitativa dos mapas com a média final. Embora não confirmados em 2013, os resultados encontrados em 2012 apontam evidências de que os mapas conceituais podem servir como outro instrumento ou parâmetro para uma avaliação mais precisa e coerente com os resultados de aprendizagem pretendidos.

Quanto à percepção dos alunos em relação ao uso dos mapas, pode-se concluir também que a maioria os considerou úteis e interessantes. Além disso, uma parcela significativa indicou ter utilizado os mapas em outras atividades e disciplinas do curso. Isso pode ser uma forte evidência de que os mapas conceituais são ferramentas que possuem potencial de uso como método de ensino e de avaliação da aprendizagem.

De modo geral, o uso dos mapas conceituais como instrumento de avaliação do processo de ensino-aprendizagem não exclui ferramentas tradicionais de avaliação. Pelo contrário, conforme parte dos resultados obtidos para a abordagem testada neste estudo, a combinação de provas e mapas conceituais como meios de avaliação pode tornar o processo mais abrangente e efetivo.

\section{Referências Bibliográficas}

ADAMS, J. Transport Planning. London, UK: Routledge \& Kegan Paul, 1981.

AUSUBEL, D. P.; NOVAK, J. D. \& HANESIAN, H. Psicologia Educacional. Rio de Janeiro: Editora Interamericana, 1980.

BERBEL, N. A. N. "A Problematização e a Aprendizagem Baseada em Problemas: Diferentes Termos ou Diferentes Caminhos?". Interface - Comunicação, Saúde, Educaşão, vol. 2, n. 2, pp. 139-154, 1998.

CORRÊA, R. R. Avaliasão Formativa: O Mapa Conceitual na Autorregulação da Aprendizagem. Dissertação (Mestrado em Educação), Centro de Educação, Comunicação e Artes, Universidade Estadual de Londrina, 2009.

CANTÚ, E. Elementos para o Fortalecimento da Mediação Docente na Educasãa Tecnológica: Aplicasãa no Ensino- Aprendizagem de Redes de Computadores. Tese (Doutorado em Engenharia Elétrica), Universidade Federal de Santa Catarina, 2005.

GOULDEN, M.; RYLEY, T. \& DINGWALL, R. "Beyond 'Predict and Provide': UK Transport, the Growth Paradigm and Climate Change". Fournal of Transport Policy Elsevier, n. 32, pp. 139-147, 2014.

LUCKESI, C. G. Avaliação da Aprendizagem Escolar: Estudos e Proposições. 22 ed. São Paulo: Cortez, 2011.

MOREIRA, M. A. \& MASINI, E. F. S. Aprendizagem Significativa: A Teoria de David Ausubel. São Paulo: Editora Moraes, 1982.

NOVAK, J. D. "The Theory Underlying Concept Maps and How to Construct Them". IHMC - Institute for Human and Machine Cognition. University of West Florida, USA, 2003. Disponível em: <http://cmap.ihmc.us/docs/ theory-of-concept-maps $>$. Acessado em mar. 2016.

PALMERO, M. L. R. "La Teoría del Aprendizaje Significativo". In: CAÑAS, A. J.; NOVAK, J. D. \& GARCÍA, F. G. (eds.). Concept Maps: Theory, Methodology, Technology - Proceedings of the First International Conference on Concept Mapping, vol. 1, pp. 535-544, 2004.

RODRIGUES DA SILVA, A. N. "A Problem-Project-Practice Based Learning Approach for Transportation Planning Education". Proceedings 
of the PBL 2010 International Conference - Problem-Based Learning and Active Learning Methodologies, São Paulo - SP (em CD-ROM). São Paulo: University of São Paulo/ Pan-American Network of Problem-Based Learning, 2010.

RUIZ-MORENO, L.; SONGSONO, C.; BATISTA, S. \& BATISTA, N. "Mapa Conceitual: Ensaiando Critérios de Análise". Ciência e Educação, vol. 13, n. 3, pp. 453-463, 2007.

SILVA JUNIOR, C. A. P. Engenharia de Transportes no Ensino de Graduação: Competências, Habilidades, Processo de Ensino-Aprendizagem e Avaliação. Tese (Doutorado em Planejamento e Operação de Sistemas de Transporte). Escola de Engenharia de São Carlos-USP. São Carlos, 2014 .

; FONTENELE, H. B. \& RODRIGUES DA SILVA, A. N. "Estilos de Ensino versus Estilos de Aprendizagem no Processo de Ensino-Aprendizagem: Uma Aplicação em Transportes". Transportes, vol. 21, n. 2, pp. 30-37, 2013. DOI: 10.4237/transportes. v21i2.686.

; \& \& . "Transportation Engineering Education for Undergraduate Students: Competencies, Skills, Teaching-Learning, and
Evaluation". Fournal of Professional Issues in Engineering Education and Practice, vol. 141, n. 3. pp. 05014006-1 05014006-9, 2015.

SOUZA, N. A. "Avaliação da Aprendizagem e Atuação Docente". Estudos em Avaliação Educacional, n. 29, pp. 149-168, 2004.

\& BORUCHOVITCH, E. "Mapas Conceituais e Avaliação Formativa: Tecendo Aproximações". Educação e Pesquisa, São Paulo, vol. 36, n. 3, pp. 795-810, 2010a.

\&__. "Mapas Conceituais: Estratégia de Ensino/Aprendizagem e Ferramenta Avaliativa". Educação em Revista, Belo Horizonte, vol. 26, n. 3, pp. 195-218, 2010b.

$\&$

"Mapa Conceitual: Seu Potencial como Instrumento Avaliativo". Pro-Posições, Campinas, vol. 21, n. 3, pp. 173-192, 2010c.

STANGE LOPES, B. J. O Mapa Conceitual como Ferramenta Avaliativa. Dissertação (Mestrado em Educação), Universidade Estadual de Londrina, 2007.

TAVARES, R. \& LUNA, G. "Mapas Conceituais: Uma Ferramenta Pedagógica na Consecução do Currículo". Principia, n. 15, pp. 110-116, 2007. 